\title{
Crayfish can grow neurons from immune cells
}

In the freshwater crayfish species Procambarus clarkia and Pacifastacus leniusculus, the production of neurons continues throughout the organisms' lives. New neurons are continually added to visual areas located in the eyestalks and to two groups of neurons in the sensory area of the brain called cell clusters 9 and 10, which contain neurons in the olfactory pathway. These new neurons appear to arise from neuronal precursors located in a niche that is loosely attached to the underside of the brain. The precursors are not self-renewing, however, raising the question of how the pool of neuronal precursors is replenished.

The importance of communication between the nervous system and the immune system is becoming more and more apparent in mammals as well as in invertebrates. Hemolymph, the arthropod equivalent of blood, contains cells called hemocytes, which underlie innate immunity in invertebrate organisms instead of blood cells. The neurogenic niche of crayfish is continually bathed in hemolymph, which is also funneled into the niche via the vascular cavity. Neuroscientist Jeanne Benton hypothesized that these hemocytes might be the source of new neuronal stem cells.

Benton and her colleagues at Wellesley College (MA) tested this idea by altering the total number of hemocytes in crayfish using an immune system factor called astakine 1 , which controls hemocyte production. As the scientists increased the number of hemocytes produced in the crayfish, they saw a corresponding increase in the number of neuronal stem cells in the niche (Dev. Cell 30, 322-333; 2014). They determined that hemocytes are attracted to the neurogenic niche in the crayfish brain via a serotonergic signaling mechanism, extending processes into the niche and integrating among niche cells within $6 \mathrm{~h}$.

In a second experiment, Benton's team extracted hemocytes from donor crayfish, labeled them with a cell marker and transfused them into receptor crayfish. Three days later, the label was observed in cells in the niche, and later, labeled cells were found

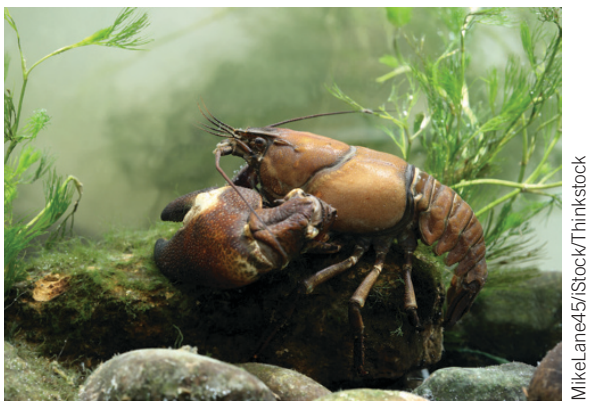

migrating away from the niche. After niche cells divided, they migrated along designated streams toward proliferation zones in cell clusters 9 and 10 , where they divide at least one more time before differentiating into neurons. The labeled cells also expressed the neurotransmitters characteristic of neurons found in this region.

The study demonstrates a natural occurrence of transdifferentiation, or the process by which one cell type turns into another cell type, which may be useful for applications in regenerative medicine.

\section{Kara Rosania}

\section{STRENGTHENING BONE IN NEUROFIBROMATOSIS TYPE 1}

Neurofibromatosis type 1 (NF1) is characterized by skeletal abnormalities such as scoliosis, fragility, fractures and pseudoarthrosis (failure of bones to fuse properly after fractures). The disorder is caused by mutations of neurofibromin, a protein that regulates cellular signaling pathways. New research from investigators at Vanderbilt University (Nashville, TN) suggests that treatment with the enzyme asfotase- $\alpha$ prevents skeletal defects in a mouse model of NF1 by increasing bone growth, mineralization and strength. Florent Elefteriou, who led the study, hopes the work will lead to treatments for people with NF1, many of whom are children. Currently, skeletal anomalies are managed by invasive and often repeated surgical intervention, which is not only painful but also limited to correction of bone abnormalities. Elefteriou and his group sought a strategy that could prevent bone defects from occurring at all. But first, they needed to understand the mechanism underlying abnormal skeletal development in the absence of neurofibromin.

Elefteriou and his colleagues studied the molecular pathology of skeletal defects in NF1 using a mouse model. They observed poor matrix mineralization in bone tissue from mice lacking neurofibromin, a condition called hyperosteoidosis, which was caused by accumulation of pyrophosphate, an inhibitor of bone mineralization. In the absence of neurofibromin, the expression of genes that promote synthesis and transport of pyrophosphate was upregulated. In addition, bone progenitor cells failed to differentiate properly into osteoblasts and did not produce alkaline phosphatase, an enzyme that normally breaks down pyrophosphate, further contributing to the accumulation of pyrophosphate and its consequent inhibition of bone mineralization.

The investigators wondered whether eliminating the accumulated pyrophosphate could improve bone mineralization. They treated NF1 model mice with asfotase- $\alpha$, a recombinant form of alkaline phosphatase that is being tested for the treatment of hypophosphatasia, another rare genetic disease affecting bone formation. Treatment with asfotase- $\alpha$ improved bone mass, mineralization and mechanical properties in the mice (Nat. Med. 20, 904-910; 2014).

Elefteriou's group also examined pseudoarthrosis samples from people with NF1 and found upregulated expression of one of the genes promoting pyrophosphate synthesis, suggesting that NF1 pathology is similar in humans and mice and, therefore, that asfotase- $\alpha$ might be effective for improving bone strength in people with the disease. Elefteriou hopes that "we can make bone stronger and better by injecting this drug, and possibly prevent fractures in patients with neurofibromatosis," he said in a news release. "It's exciting that instead of fixing the bones after they break, we might have a drug now to prevent the fractures."

Monica Harrington 